\title{
A PROSPECTIVE STUDY ON JUVENILE PRIMARY HYPERPRATHYROIDISM POPULATION
}

\section{F. Saponaro, F. Cacciatore, E. Vignali, A. Picone, S. Chiavistelli, A. Meola, E. Pardi, S. Borsari, C. Marcocci, F. Cetani UNIT OF ENDOCRINOLOGY 2, UNIVERSITY OF PISA, ITALY}

\section{INTRODUCTION}

Primary hyperparathyroidism (PHPT) is the third most common endocrine disorder in adults and its typical presentation is an incidentally discovered asymptomatic hypercalcemia. It occurs in patients between the age of 50 and 60 years, with an annual incidence of 30 cases per 100,000 and females/males ratio of approximatly $3: 1$.

PHPT is uncommon in young people and children.

Since the first case described in 1930 by Pemberton and Geddie, studies on Juvenile PHPT (J-PHPT) are few, mostly retrospective and on limited numbers.

The estimated incidence of J-PHPT is $2-5$ in 100,000 and without difference of sex. J-PHPT seems to be clinically symptomatic more frequently than the adult form and typically presents with signs or symptoms of hypercalcemia, skeletal complications, and/or nephrolithiasis. It's still controversial if this finding is due to a real biologic difference that accounts for the distinct features between PHPT in young and older patients or it's a bias due to infrequent biochemical testing of children and adolescents.

\section{AIM OF WORK}

The aim of the study was to evaluate the clinical, biochemical, densitometric, hystological characteristics and outcome in patients with J-PHPT, comparing sporadic (S) and familial (F) J-PHPT.

\section{MATERIALS AND METHODS}

PATIENTS It's a monocentric prospective study at a referral center. Two hundreds patients/year were evaluated in our center in a period of 9 years (2006-2014) and 154 patients with J-PHPT, namely with age of $\leq 40$ years (range 12-40 years), were enrolled in the present study. Patients were fully evaluated at diagnosis and at the last follow-up visit (median follow-up 2 years) by: clinical evaluation, biochemical data collection, densitometric analysis, histology after parathyroidectomy (PTX). Patients were classified in F and S J-PHPT on the basis of i) familial clinical history, ii) absence of familial PHPT features at physical examination; iii) neuroendocrine markers, calcitonin, pituitary hormones in the normal range at diagnosis and last follow-up visit; iv) serum calcium in first-degree relatives in the normal range. One hundred-twelve patients had SJ-PHPT and 42 patients FJ-PHPT. The latter group included 31 patients with Multiple Endocrine Neoplasia Type 1 Syndrome (MEN1) and 11 with Familial Isolated Hyperparathyroidism (FIHP).

GENETIC ANALYSIS DNA of index cases of MENI and FIHP families was purified from periferical blood with a commercial kit (Qiagen Blood Minikit) and the entire coding regions and splice sites junctions of MEN1 gene, were PCR-amplified and a sequencing reaction was performed. In FIHP patients, negative to MENI testing, CASR, CDC73, CDKNIB and AIP genes were also analyzed as above described.

\section{RESULTS}

Clinical data from all patients are reported in Figure 1. For MEN 1 patients we also reported the distribution of pituitary, neuroendocrine and cutaneous lesions (Fig. 1).

Figure 1

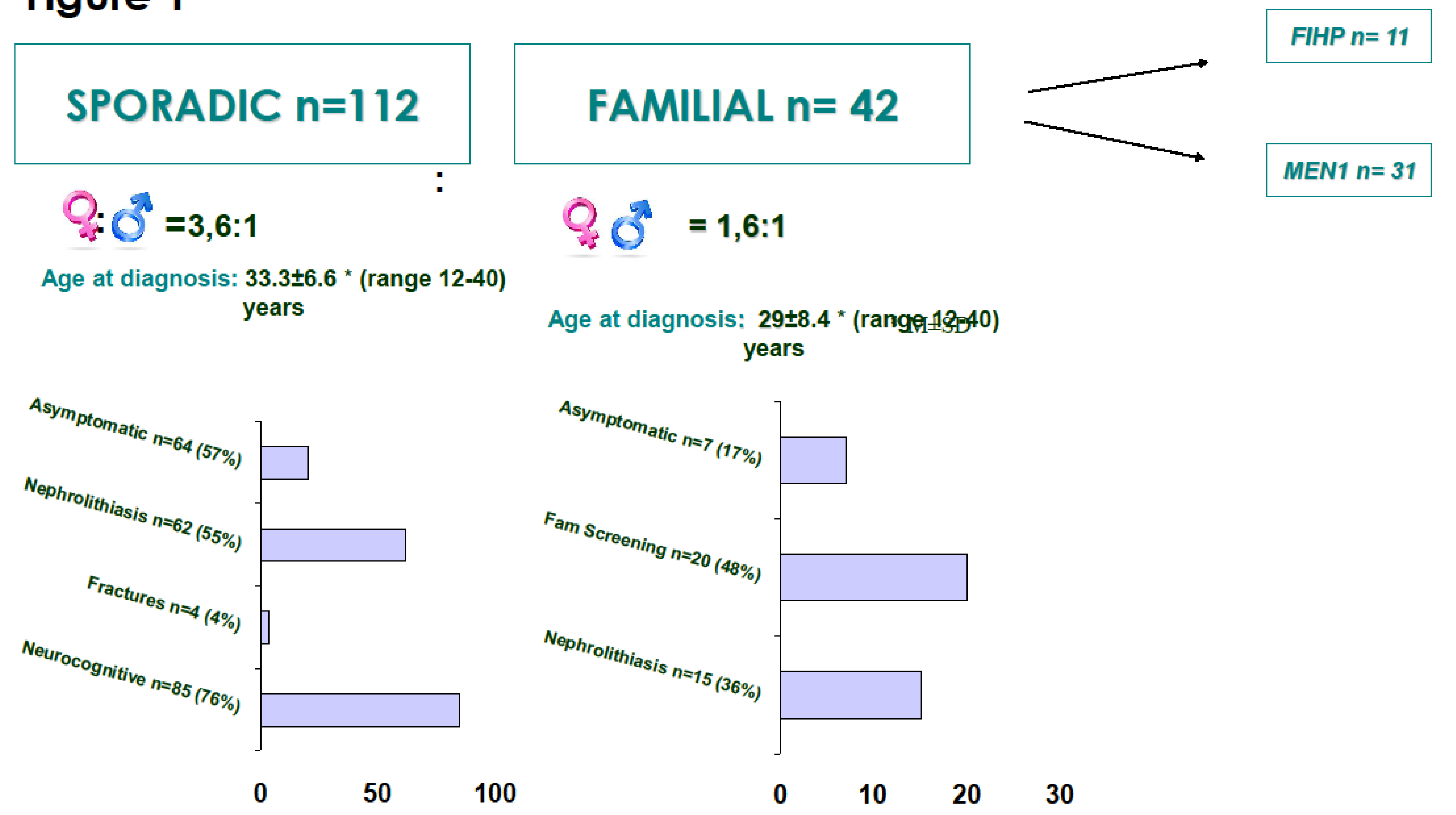

Ninety Sporadic (S) J-PHPT and 27 Familial (F) J-PHPT underwent PTx. The histology showed in SJ-PHPT and FJ-PHPT a single adenoma in 85 and 7 patients, hyperplasia in 2 and 19 , white cervicotomy in 1 and 1, respectively. In 2 S J-PHPT patients histology showed a parathyroid carcinoma All but 7 (9.6\%) patients with SJ-PHPT were cured. The patients who had persistence/recurrence of PHPT included one with white cervicotomy, one with hyperplasia and 5 with single adenoma. Patients who did not undergo PTx, had a stable disease. All but 13 (31\%) patients with F J-PHPT were cured (Fig. 2).

Figure 2
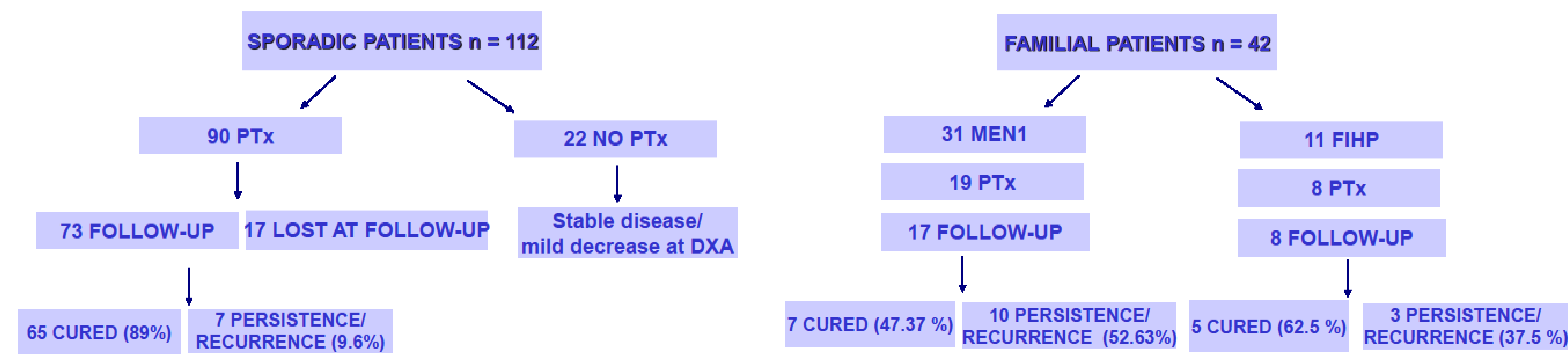

In both groups a more severe disease in term of serum Ca++ (SJ-PHPT $p=0.039$, FJ-PHPT $p=0.027$ ), PTH (S J-PHPT $p=0.045, F$ J-PHPT $p=0.7$ ) and $3^{\circ}$ distal radius $Z$ score (Sporadic $p=0.001$, Familial $p=0.002$ ) was observed in males compared with females. In contrast, age was not significantly correlated with the severity of disease in term of $\mathrm{Ca}++(p=0.4)$, PTH $(p=0.2)$ and $Z$ score in both groups ( $\mathrm{p}=0.7$ ) In both groups basaline serum Ca++ was significantly associated with PTH levels and severity of bone disease (lumbar, hip and $3^{\circ}$ distal radius $Z$ score) $(p<0.001)$.

When the patients, in the overall group, were stratified for age, $\leq 25(n=31)$ and $>25$ years $(n=123)$, serum calcium levels was statistically higher ( $p$ $=0.024)$ and $3^{\circ}$ distal radius $Z$ score significantly lower $(p=0.009)$ in younger patients.

In Figure 3 we reported the results of genetic analysis of MEN1 and FIHP patients.

\section{Figure 3}
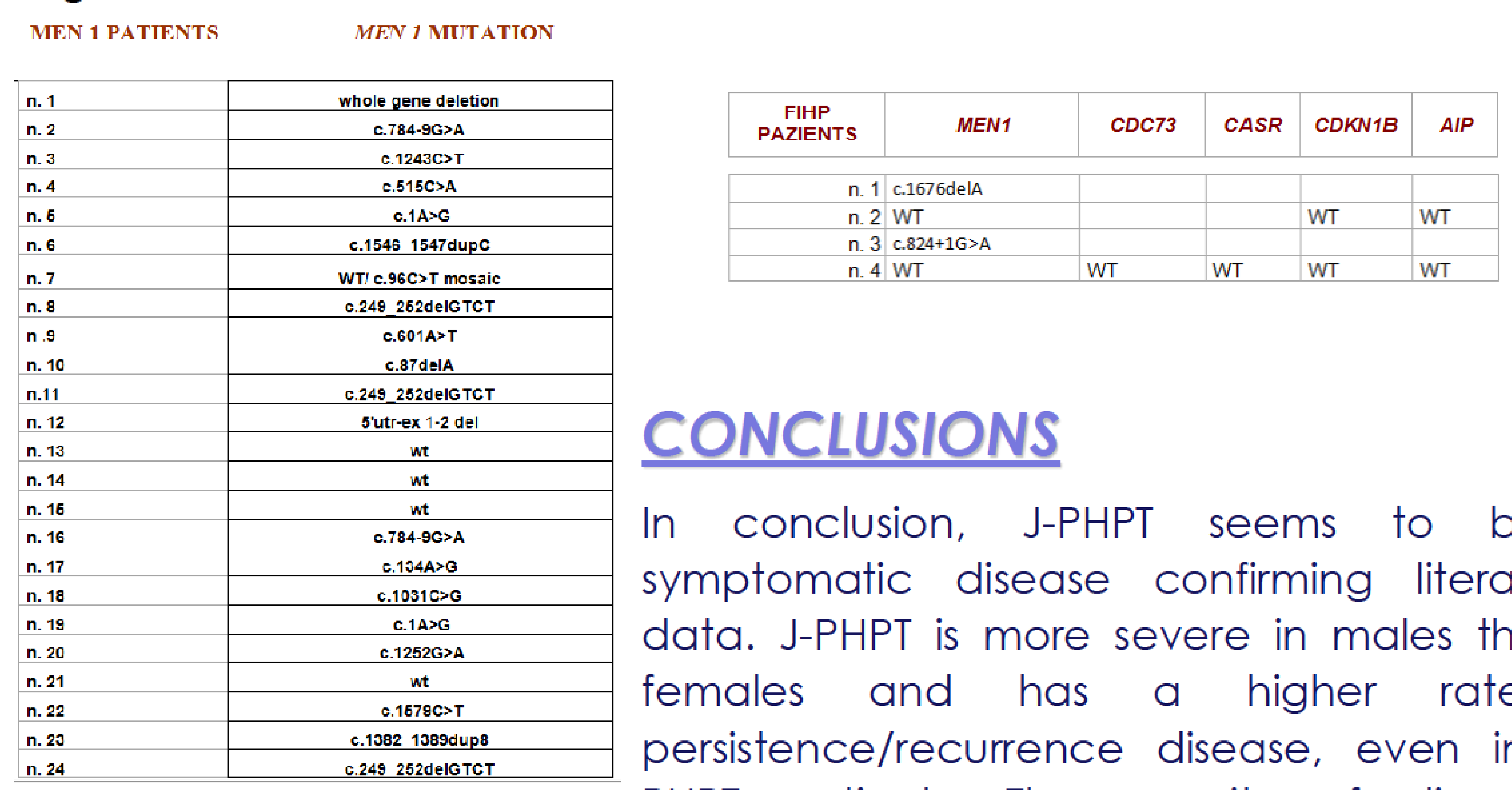

\section{CONCLUSIONS}

In conclusion, J-PHPT seems to be a symptomatic disease confirming literature's data. J-PHPT is more severe in males than in females and has a higher rate of persistence/recurrence disease, even in S-JPHPT patients. The severity of disease is independ from age and the clinical characteristics are similar between Sporadic and Familial patients, .

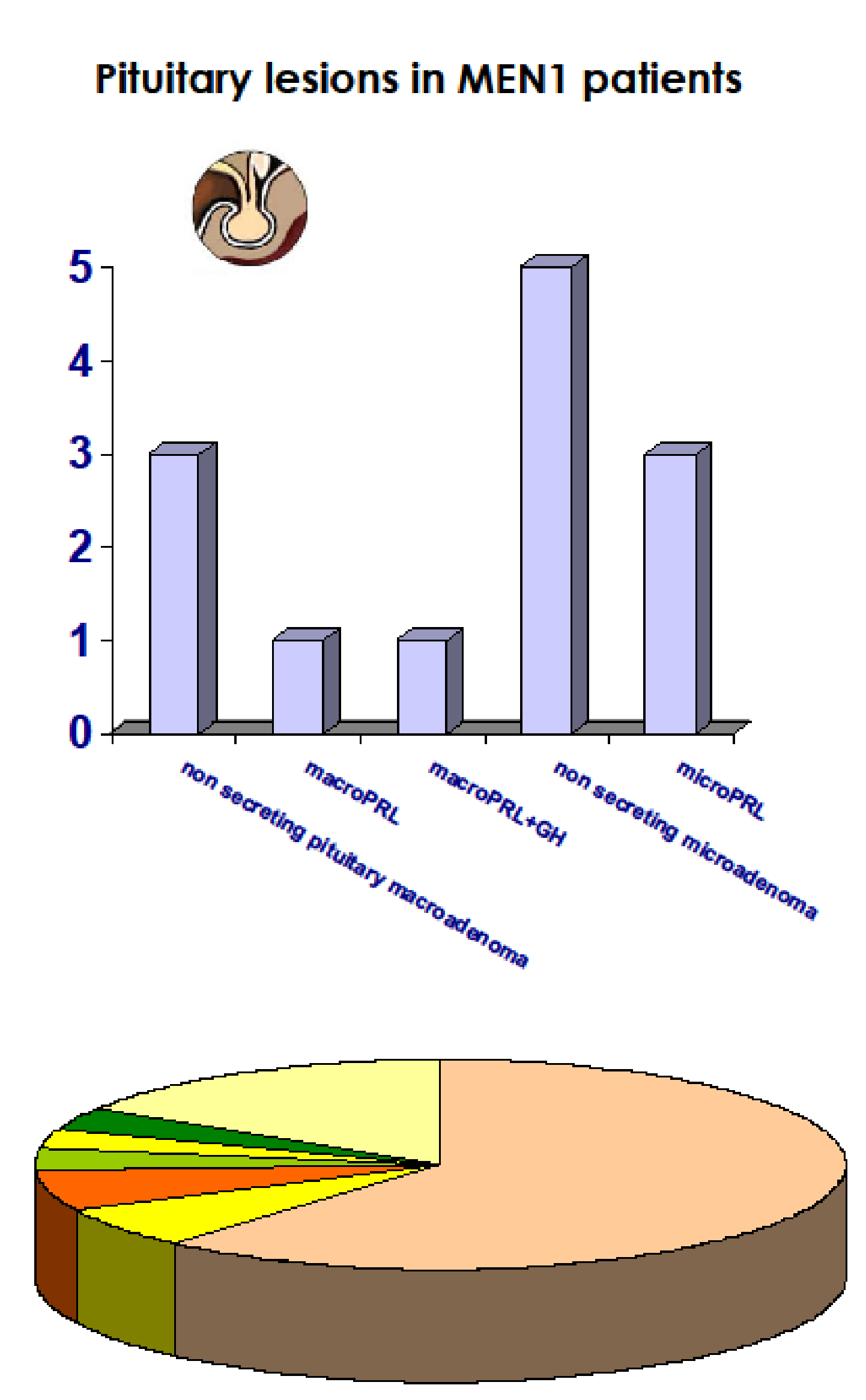

